\title{
Human papillomavirus DNA detection in adults and elderly patients with head and neck cancer
}

\author{
Alessandra Sbegue (IC), Stella V. S. Torres (PG), Sandra H. A. Bonon (PG), Sandra C. B. Costa (PQ).
}

\begin{abstract}
The relationship between human papillomavirus and head and neck cancers has been demonstrated in the literature, and this study aims to demonstrate the prevalence of this virus in head and neck cancers of twenty-seven adults and elderly patients attended in the hospital of clinics Unicamp Campinas, in 2014.
\end{abstract}

Key words: Human papillomavirus, head and neck cancer, elderly.

\section{Introduction}

Virus has been associated as causative agents for a large proportion of various human

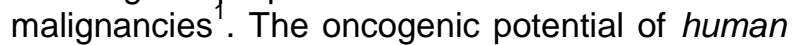
papillomavirus (HPV) and its possible role in the development of malignant conditions, in particular cancer of head and neck has been described.

This study evaluated the presence of HPV DNA in fresh biopsies of tissues surgically removed of twenty-seven adults and elderly patients, aged 45 to 75 years.

\section{Results and Discussion}

Fresh biopsies from 16 patients with head and neck cancer and 11 fresh biopsies from a healthy control group were studied. DNA extraction was made and nested PCR technique was used for DNA detection in lesions.

HPV occurred in 8/16 (50\%) cancer biopsies and only one patient in control group was positive $(9,1 \%)$.

Worldwide, cancer centers report that virus is responsible for between $45 \%$ and $90 \%$ of oropharyngeal cancers ${ }^{2}$. According to a metaanalysis conducted by the University of Birmingham, UK, oropharyngeal cancer (OPC) associated with HPV quadrupled in number over a period of 10 to 15 years. In contrast, the relatively low prevalence of HPV in non-OPC remains unchanged $^{3}$. More than $90 \%$ of HPV-related oropharyngeal cancers are caused by HPV-16, a particularly dangerous strain and the main cause of cervical cancer. In other study, D'Souza et al. ${ }^{4}$ showed the association was similarly increased among subjects with oral HPV-16 infection, regardless of their tobacco and alcohol use. By contrast, tobacco and alcohol use increased the association with oropharyngeal cancer primarily among subjects without exposure to HPV-16. Oral HPV infection is strongly associated with oropharyngeal cancer among subjects with or without the established risk factors of tobacco and alcohol use.

Chart 1. Positive and negative samples of fresh tissue of the groups of head and neck cancer and control, using the technique of nested-PCR for the detection of human papillomavirus DNA.

\begin{tabular}{|c|c|c|}
\hline Groups & $\begin{array}{c}\text { Positive } \\
\text { samples }\end{array}$ & $\begin{array}{c}\text { Negative } \\
\text { samples }\end{array}$ \\
\hline Cancer group $(n=16)$ & 8 & 8 \\
\hline Control group $(n=11)$ & 1 & 10 \\
\hline p-value & \multicolumn{2}{|c|}{0,0417} \\
\hline
\end{tabular}

Fischer's test: $p$-value $<0,005-95 \% \mathrm{Cl}$.

\section{Conclusions}

HPV is related to the development of head and neck cancer, and our study found a higher prevalence in subjects in the cancer group compared to the control group, corroborating with the findings of other authors.

\section{Acknowledgement}

The authors wish to thank the University of Campinas/UNICAMP for the support. This study was also supported by a grant \#2014/19446-1, from São Paulo Research Foundation (FAPESP).

\footnotetext{
${ }^{1}$ Persing DH, Prendergast FG. Infection, Cancer and the immune response. In: The Infectious Etiology of Chronic Diseases: Defining the Relationship, Enhancing the Research, and Mitigating the Effects- Workshop Summary 2004, 3: 154-73.

${ }^{2}$ Scudellari, M. HPV: Sex, cancer and a virus. Nature. 2013; 503 : 330-32.

${ }^{3}$ Mehanna H, Beech T, Nicholson T, El-Hariry I, McConkey C, Paleri V, Roberts S. Prevalence of human papillomavirus in oropharyngeal and nonoropharyngeal head and neck cancer systematic review and meta-analysis of trends by time and region. Head Neck. 2013;35(5):747-55.

${ }^{4}$ D'Souza G, Kreimer AR, Viscidi R, Pawlita M, Fakhry C, Koch WM, Westra WH, Gillison ML. Case-control study of human papillomavirus and oropharyngeal cancer. N Engl J Med. 2007;356(19):1944-56..
} 